\title{
A REFLECTION ON THE USEFULNESS AND EFFECTIVENESS OF THE MODERN RISK-ORIENTED AUDIT IN CHINA
}

Qianshan George Gao, Shih-Jen Kathy Ho, and Audrey Agnello

The BRC Academy Journal of Business 5, no. 1 (2015): 19-32.

http://dx.doi.org/10.15239/j.brcacadjb.2015.04.01.ja02

\section{WEB APPENDIX}

http://dx.doi.org/10.15239/j.brcacadjb.2015.04.01.wa02 
Table 1: Audit Fees Analysis during 2004-2008

( in ten thousand RMB)

\begin{tabular}{|c|c|c|c|c|}
\hline C-Year & $\mathbf{N}^{*}$ & $\begin{array}{c}\text { Audit Fees } \\
\text { Total }\end{array}$ & $\begin{array}{c}\text { Audit Fees } \\
\text { per Company }\end{array}$ & $\begin{array}{c}\text { Audit Fees Increase } \\
\text { per Company }\end{array}$ \\
\hline 2004 & 1212 & 75,890 & 62.6 & - \\
\hline 2005 & 1177 & 75,479 & 64 & $2.24 \%$ \\
\hline 2006 & 1094 & 79,010 & 72 & $12.5 \%$ \\
\hline 2007 & 994 & 78,290 & 78.8 & $9.44 \%$ \\
\hline 2008 & 1116 & 88,960 & 79.7 & $1.14 \%$ \\
\hline
\end{tabular}

*The sample size changes because some companies did not disclose their audit fees.

Table 2: Audit Opinions for 2005-2008（n=1,331 overall）

\begin{tabular}{|l|c|c|c|c|c|c|c|}
\hline Year & MO* & MOE & UO & UOE & DO & RUOE & RNO \\
\hline 2005 & 42 & 16 & 1172 & 73 & 28 & $6.23 \%$ & $13.57 \%$ \\
\hline 2006 & 28 & 8 & 1192 & 75 & 28 & $6.30 \%$ & $11.66 \%$ \\
\hline 2007 & 13 & 1 & 1212 & 90 & 15 & $7.43 \%$ & $10.64 \%$ \\
\hline 2008 & 15 & 0 & 1223 & 76 & 17 & $6.21 \%$ & $8.83 \%$ \\
\hline
\end{tabular}

* $\mathrm{MO}=$ modified opinion; $\mathrm{MOE}=$ modified opinion with emphasis - of matter paragraphs $\mathrm{UO}=$ unmodified opinion; $\mathrm{UOE}=$ unmodified opinion with explanatory paragraph; $\mathrm{DO}=$ disclaimer of opinion; RUOE = ratio of UOE to all reports; RNO =ratio of nonstandard opinion to all reports. 
Table 3: Companies with Unmodified Opinions (UO)

for their Mid-Term Reports in 2008

\begin{tabular}{|c|c|c|c|}
\hline Company Code & $\begin{array}{c}\text { EPS for the First } \\
\text { Half }\end{array}$ & $\begin{array}{c}\text { EPS for Annual } \\
\text { Report }\end{array}$ & $\begin{array}{c}\text { Date of Issue for Audit } \\
\text { Report }\end{array}$ \\
\hline 000001 & 0.71 & 0.26 & $8 / 20$ \\
\hline 000050 & 0.11 & 0.024 & $8 / 25$ \\
\hline 002002 & -0.12 & -1.01 & $8 / 28$ \\
\hline 600058 & 1.64 & 0.85 & $8 / 28$ \\
\hline 600022 & 0.78 & 0.55 & \\
\hline
\end{tabular}

